\title{
Method for determination of interaction between a two-wheeled self-balancing vehicle and its rider
}

\author{
M. Ciężkowski \\ Bialystok University of Technology, Wiejska 45C,15-351 Bialystok, Poland, E-mail: mciezkowski@gmail.com
}

cross $^{\text {ref }}$ http://dx.doi.org/10.5755/j01.mech.22.5.13315

\section{Introduction}

A two-wheeled self-balancing vehicle (the commercial version is called the Segway ${ }^{\mathrm{TM}}$ ) is a device in which acceleration of a vehicle is controlled by an appropriate balancing of the rider's body. The rider's interaction with the vehicle's platform causes the vehicle to lean; the on-board computer records it and on that basis controls the vehicle's motors. The rider-vehicle interaction consists in applying torque by the rider to the vehicle's platform, causing its rotation. The control method of such a system is very similar to the problem of stabilizing the two-wheeled inverted pendulum, see for example [1-3]. There are many papers describing the theory of TWSBV (a two-wheeled self-balancing vehicle), where the main attention is focused on the problem of stability of an already leaning vehicle [4-8], and a mathematical model of the vehicle is approximated by a two-wheeled inverted pendulum. This approach is correct if the model is used only to determine the stability control of the vehicle in the upright position. Such a model has also been used in this work, but only in order to determine the motor control. At this point the question arises whether the model of a two-wheeled selfbalancing vehicle with a rider can be completely approximated by two-wheeled inverted pendulum model. The answer is no, because a model like that does not include interaction between the vehicle and its rider, and the inverted pendulum cannot unbalance itself from the equilibrium position, what is the primary goal of the TWSBV rider. The rider is the one who decides how fast and in what direction the vehicle is moving and he does it by properly balancing its body. It can be concluded that the main signal controlling the vehicle does not act directly on the motors, but is the result of interaction between the vehicle and its rider. To consider this interaction in the model of the vehicle with the rider, at least one additional degree of freedom that describes the user's position relative to the vehicle must be included in the mathematical description of the system. In other words, the rider of the vehicle, together with the platform on which it stands, cannot be described by one rigid body system. In 2013, Mohtasib A.M. and Shawar M.H. in [9] presented a model of the system which contained the rider-vehicle interaction. The value of this interaction, however, was determined arbitrarily.

The main objective of this paper is to present a method of determining a quantitative and qualitative description of interaction between the two-wheeled selfbalancing vehicle and its rider. It should be noted that this is a new topic in research, because such interactions have not been studied previously, and inclusion of this interaction into the mathematical model of the rider-vehicle system makes it more realistic. To achieve this goal the exper- iment using a self-designed vehicle was performed (Section 2). The next step was to develop a mathematical model of the system, including the rider-vehicle interaction (Section 3). One of the most important things was to hypothesize a qualitative mathematical form of interaction (Section 4). The final step was to determine the unknown interaction based on the comparison of experimental data with the computer simulation results (Section 5). The general form of interaction between the vehicle and its rider is given in Section 6. Preliminary results are presented in [10].

\section{Experimental results}

To collect the experimental data, the self-designed vehicle was used. The vehicle is shown in Fig. 1. It consists of a platform on which the rider stands, a handlebar to change the direction of travel and wheels driven by electric motors located under the platform of the vehicle. To estimate the inclination angle of the platform and its angular velocity the inertial measurement unit was used. Velocity of the vehicle was calculated using an incremental quadrature encoder mounted on motor axes.
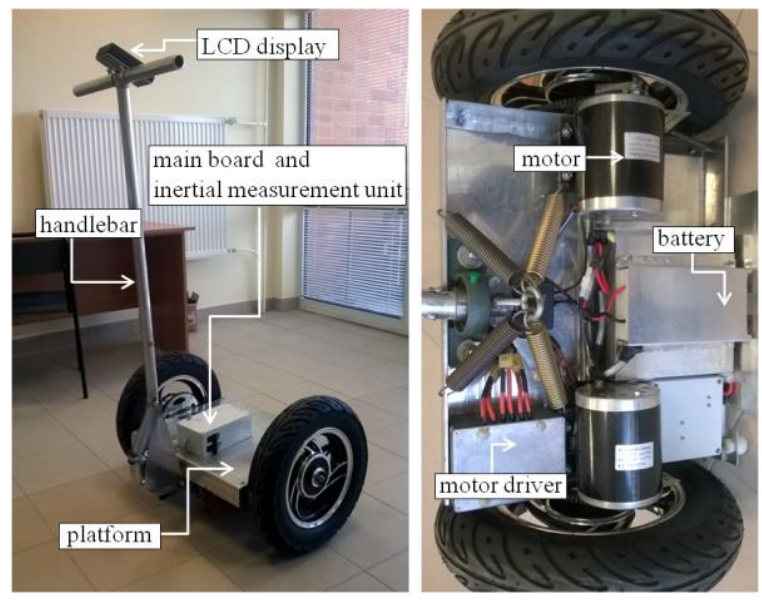

Fig. 1 Two-wheeled self-balancing vehicle

The experiment consisted of a straight line acceleration from the zero vehicle speed to the desired final velocity $V_{T}$ in time $T$, and a simultaneous measurement of selected variables of the state vector. The measured variables were the velocity of the vehicle and the angle of inclination of the platform. The data was logged into the microcontroller's memory with a frequency of $10 \mathrm{~Hz}$. There were thirty experiments performed for various values of the final velocity. The outcome of one of these is presented in Fig. 2. The solid lines describe the measurement data ( $\alpha$-platform inclination, $V$ - vehicle velocity). The dashed 
lines come from the polynomial interpolation of measurement data derived by Hermite interpolation [11]. These interpolation functions will be used in further analysis.

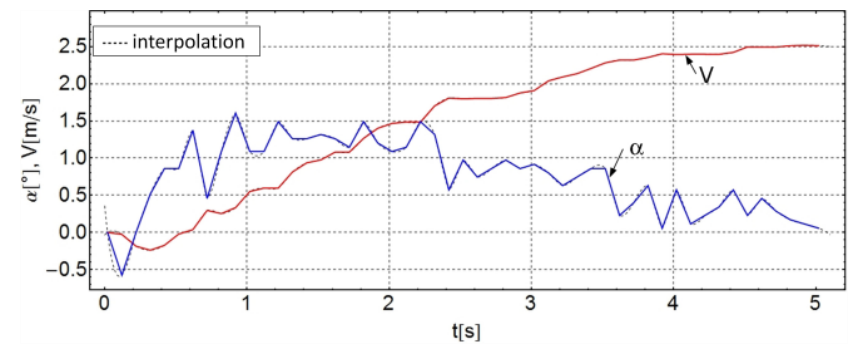

Fig. 2 Experimental results for experiment no. 1

\section{Mathematical model}

To enable interaction between the rider and vehicle, an extra degree of freedom was added to the system, enabling change of the user's position relative to the platform. This additional degree of freedom is the ankle $\phi$ (Fig. 3), because users of this type of vehicles mainly bend at the ankle joint as seen from observation.
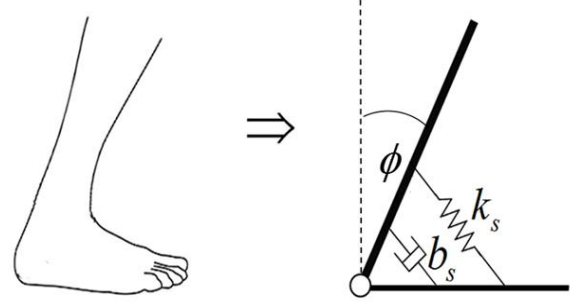

Fig. 3 Ankle joint mode

Torque generated in the ankle joint consists of a passive part (instinctive, not user controlled) and an active part (completely under the user control):

$$
M_{s}=T_{u}-k_{s} \phi-b_{s} \dot{\phi}
$$

where $T_{u}$ is a torque active part, and $-k_{s} \phi-b_{s} \dot{\phi}$ is a passive part. The passive part of total torque $M_{s}$ was approximated by a damped harmonic oscillator model $[12,13]$. The active part of torque is unknown and its designation is the main goal.

The model of the vehicle platform was approximated by a perfectly rigid body of known mass and the moment of inertia. The rider's model was approximated by a perfectly rigid body of known mass, the moment of inertia and the centre of gravity position. The rider is coupled to the vehicle by the ankle joint. The wheels of the vehicle were described by a rigid body of known parameters. The system has three degrees of freedom: $\alpha$-platform inclination, $\beta$ - user inclination, and $\theta$ - wheel rotation angle (and thus the position of the vehicle, because the movement takes place without slipping). As you can see the model does not take account of the changing direction. The vehicle-rider interaction, which results in a change of direction, is simply a deflection of the handlebar, so it is not very interesting. In addition, the experiment concerned the straight line changing speed, and therefore a more complex model is not necessary.

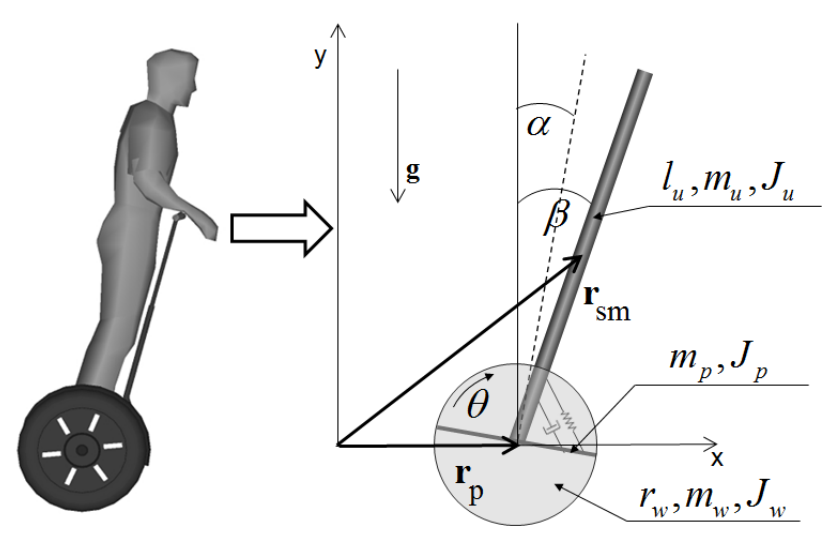

Fig. 4 Physical model of vehicle

The Table 1 contains values of the system parameters.

Table 1

The vehicle and the rider's parameters

\begin{tabular}{|l|c|c|c|}
\hline \multicolumn{1}{|c|}{ Parameter } & Symbol & Value & Unit \\
\hline Mass of the platform & $m_{p}$ & 26.7 & $\mathrm{~kg}$ \\
\hline $\begin{array}{l}\text { Moment of inertia of the } \\
\text { platform }\end{array}$ & $J_{p}$ & 0.712 & $\mathrm{kgm}^{2}$ \\
\hline Mass of the wheel & $m_{w}$ & 5.18 & $\mathrm{~kg}$ \\
\hline Moment of inertia of the wheel & $J_{w}$ & 0.096 & $\mathrm{kgm}^{2}$ \\
\hline Mass of the rider & $m_{u}$ & 80 & $\mathrm{~kg}$ \\
\hline Moment of inertia of the rider & $J_{u}$ & 13.1 & $\mathrm{kgm}^{2}$ \\
\hline Wheel radius & $r_{w}$ & 0.215 & $\mathrm{~m}$ \\
\hline Rider's center of gravity & $l_{u}$ & 0.98 & $\mathrm{~m}$ \\
\hline Ankle elasticity coefficient & $k_{s}$ & 850 & $\mathrm{Nm} / \mathrm{rad}$ \\
\hline Ankle damping coefficient & $b_{s}$ & 350 & $\mathrm{Nms} / \mathrm{rad}$ \\
\hline Motor torque coefficient & $k_{m}$ & 0.53 & $\mathrm{Nm} / \mathrm{A}$ \\
\hline Motor SEM coefficient & $k_{e}$ & 0.53 & $\mathrm{Vs} / \mathrm{rad}$ \\
\hline Motor viscosity coefficient & $k_{w}$ & 0.018 & $\mathrm{Nms} / \mathrm{rad}$ \\
\hline Motor coil resistance & $R$ & 0.21 & $\Omega$ \\
\hline Motor gear ratio & $n$ & 7.27 & \\
\hline
\end{tabular}

The system has been described by the Lagrange formalism, see [14]. The position of the platform is described by a vector:

$$
r_{p}=\left(\theta r_{w}, 0\right)
$$

Describing the position of the platform by angle $\theta$ follows from the assumption that the motion takes place without slipping. The position of the rider's center of mass is:

$$
r_{s m}=r_{p}+l_{u}(\sin \beta, \cos \beta)=\left(\theta r_{w}+l_{u} \sin \beta, l_{u} \cos \beta\right)
$$

The kinetic energy of the rider is the sum of the translational kinetic energy of the center of mass and the angular kinetic energy about an axis passing through its center of mass and parallel to horizontal axis of the wheels: 


$$
\begin{aligned}
T_{1} & =\frac{1}{2} m_{u} \dot{r}_{s m}{ }^{2}+\frac{1}{2} J_{u} \dot{\beta}^{2}=\frac{1}{2} J_{u} \dot{\beta}^{2}+\frac{1}{2} \dot{\theta}^{2} m_{u} r_{w}^{2}+ \\
& +\frac{1}{2} \dot{\beta}^{2} l_{u}{ }^{2} m_{u}+\dot{\beta} \dot{\theta} l_{u} m_{u} r_{w} \cos \beta .
\end{aligned}
$$

The kinetic energy of the platform takes the form:

$$
T_{2}=\frac{1}{2} J_{p} \dot{\alpha}^{2}+\frac{1}{2} \dot{\theta}^{2} m_{p} r_{w}^{2} .
$$

The kinetic energy of the wheels is:

$$
T_{3}=J_{w} \dot{\theta}^{2}+m_{w} r_{w}^{2} \dot{\theta}^{2}
$$

Potential energy of the rider and the spring modelling the ankle joint is:

$$
V=m_{u} g l_{u} \cos \beta+\frac{1}{2} k_{s}(\beta-\alpha)^{2} .
$$

The Lagrangian of the vehicle-rider system takes the form:

$$
L=T_{1}+T_{2}+T_{3}-V
$$

Using Eqs. (4)-(8) and taking into account nonconservative forces from the motors and the ankle, the Euler-Lagrange equations take the form:

$$
\left\{\begin{array}{l}
\frac{d}{d t} \frac{\partial L}{\partial \dot{\theta}}-\frac{\partial L}{\partial \theta}=2 M_{m} ; \\
\frac{d}{d t} \frac{\partial L}{\partial \dot{\alpha}}-\frac{\partial L}{\partial \alpha}=-2 M_{m}-b_{s}(\dot{\alpha}-\dot{\beta})-T_{u} \\
\frac{d}{d t} \frac{\partial L}{\partial \dot{\beta}}-\frac{\partial L}{\partial \beta}=b_{s}(\dot{\alpha}-\dot{\beta})+T_{u},
\end{array}\right.
$$

where the motor torque: $M_{m}=\frac{k_{m}}{R}\left(U+k_{e}\left(\frac{\dot{\alpha}}{n}-\dot{\theta}\right)\right)-k_{w} \dot{\theta}$. The solution of Eq. (9) are the equations of motion of the system:

$$
\begin{aligned}
& \ddot{\alpha}=- \frac{(\dot{\alpha}-\dot{\beta}) b_{s}+2 M_{m}+(\alpha-\beta) k_{s}+T_{u}}{J_{p}} ; \\
& \ddot{\beta}=-\frac{-\left(2 J_{w}+r_{w}^{2}\left(m_{p}+m_{u}+2 m_{w}\right)\right)\left((\dot{\alpha}-\dot{\beta}) b_{s}+(\alpha-\beta) k_{s}+T_{u}\right)+2 M_{m} l_{u} m_{u} r_{w} \cos \beta}{a}- \\
& \ddot{\theta}=\frac{-l_{u} m_{u} r_{w}\left((\dot{\alpha}-\dot{\beta}) b_{s}+(\alpha-\beta) k_{s}+T_{u}\right) \cos \beta+2 M_{m}\left(J_{u}+l_{u}^{2} m_{u}\right)}{a}+ \\
& \\
&+\frac{-g l_{u}^{2} m_{u}^{2} r_{w} \sin \beta \cos \beta+\dot{\beta}_{w}^{2}\left(-\left(m_{p}+m_{u} m_{u} r_{w} \sin \beta\left(J_{u}+l_{u}^{2} m_{u}\right)\right.\right.}{a},
\end{aligned}
$$

where $a=r_{w}^{2}\left(J_{u}\left(m_{p}+m_{u}+2 m_{w}\right)+l_{u}^{2} m_{u}\left(m_{p}+m_{u} \sin ^{2} \beta+2 m_{w}\right)\right)+2 J_{w}\left(J_{u}+l_{u}^{2} m_{u}\right)$.

To determine the motor control of the vehicle, the state feedback control has been used. This feedback is a linear function of the measured variables of the state vector, such as: $\alpha$ - platform inclination, $\dot{\alpha}$ - angular velocity of the platform, $\dot{\theta}$ - angular velocity of the wheels. Therefore, the function of voltage $U$ applied to motors takes the form: $U=k_{1} \alpha+k_{2} \dot{\alpha}+k_{3} \dot{\theta}$. Coefficients $k_{1}$ and $k_{2}$ were calculated using the LQR regulator, see for example [15, 16]. The LQR controller requires a full state feedback, but variable $\beta$ (user inclination) is not measured. Therefore, to apply the LQR control, the state vector of the system must be reduced. To achieve this, the mathematical model of the vehicle must be approximated by a two-wheeled inverted pendulum (as it was mentioned in the introduction). The reduction is based on the assumption that $\alpha=\beta$. The nominal values of the parameters $k_{1}$ and $k_{2}$ are as follows: $k_{1}=190 \frac{\mathrm{V}}{\mathrm{rad}}, \quad k_{2}=20 \frac{\mathrm{Vs}}{\mathrm{rad}}$. The parameter $k_{3}$ is determined from the equation of the motor torque, assuming that the linear velocity of the vehicle does not change when the angle of inclination of the platform is equal to zero. According to Newton's First law, assuming that $\dot{\alpha}=0$, the motor torque takes the form:

$$
M_{m}=k_{m} \frac{U+k_{e}(-\dot{\theta})}{R}-k_{w} \dot{\theta}=0 .
$$

According to the Eq. (11), the electric voltage is equal to: $U=\left(k_{e}+R k_{w} / k_{m}\right) \dot{\theta}$. Substituting the numerical values of parameters presented in Table 1 , the voltage has the following value: $U=0,537 \dot{\theta}$, therefore, $k_{3}=0.537$.

Finally, the function of voltage $U$ applied to motors takes the form, see [17]:

$$
U=190 \frac{\mathrm{V}}{\mathrm{rad}} \alpha+20 \frac{\mathrm{Vs}}{\mathrm{rad}} \dot{\alpha}+0.537 \frac{\mathrm{Vs}}{\mathrm{rad}} \dot{\theta} .
$$

The investigated system is not observable, therefore determination of the unknown torque $T_{u}$ directly from the model Eq. (10) is not possible. The solution of the problem is to find a mathematical form of the rider - vehicle interaction $T_{u}$, so that the computer simulation results using the proposed model (10) are consistent with the experimental results (according to the compliance criterion). 
In the next sections of this paper the solution method of this problem will be presented.

\section{Hypothesis about interaction form}

The main task of the rider is to obtain the desired vehicle speed, so torque $T_{u}$ should depend on the desired speed and the actual speed of the vehicle. While driving, the rider can change the elasticity and damping coefficients of the ankle joint by a suitable tension of muscles. According to these assumptions it can be hypothesized that torque $T_{u}$ takes the form:

$$
T_{u}=C_{1}\left(\dot{\theta}_{S E T}-\dot{\theta}\right)+C_{2}(\alpha-\beta)+C_{3}(\dot{\alpha}-\dot{\beta}),
$$

where $\dot{\theta}_{S E T}$ - desired vehicle speed $\left(\dot{\theta}_{S E T}=V_{T} / r_{w}\right), C_{1}$, $C_{2}, C_{3}$ - unknown parameters.

The verification method of this hypothesis is based on a numerical solution of the mathematical model of the vehicle (10), taking into account torque $T_{u}$ described by (13). The purpose of the numerical analysis is to find the matching function $\varphi=f\left(y\left(C_{1}, C_{2}, C_{3}\right), \hat{y}\right)$, where $y$ describes the time evolution of the system obtained from the numerical solution of the equations (10), and $\hat{y}$ describes the experimental data approximated by Hermite interpolation. The parameters $C_{1}, C_{2}$ and $C_{3}$, for which function $\varphi$ has a maximum are the sought coefficients of Eq. (13) for the current tested experiment.

\section{Matching results}

In order to compare the experimental data with the computer simulation results, the matching function $\varphi$ was defined based on the normalized root mean square error (NRMSE) between the experimental data and simulation data. The normalized root mean square error can be achieved by normalizing the RMSE value to the range of the observed data, see for example [18]. This function takes the form:

$$
\varphi=1-N R M S E,
$$

where $N R M S E=\frac{R M S E}{\hat{y}_{\max }-\hat{y}_{\min }}, \hat{y}_{\max }$ and $\hat{y}_{\min }$ are respectively the maximum and minimum values measured in the experiment. Due to the fact that the angle of inclination of the platform measured in the experiment is distorted by the platform vibration ( $\alpha$ plot in Fig. 2), the matching function $\varphi$ was defined based on the vehicle velocity ( $V$ plot in Fig. 2). Thus, the aim for different parameters $C_{1} C_{2}, C_{3}$, is to determine the matching function:

$$
\varphi=f\left(V\left(C_{1}, C_{2}, C_{3}\right), \hat{V}\right),
$$

where $V$ is the vehicle velocity function obtained from the simulation of model Eq. (10) $\left(V=\dot{\theta} r_{w}\right)$, and $\hat{V}$ is the Hermite interpolation function of experimental measured velocity. The matching function (15) was determined by the use of the numerical solution of the system Eq. (10) in the nodes of the mesh of parameters $C_{1} C_{2}, C_{3}$.
An appropriate selection of mesh parameters $C=\left(C_{1}, C_{2}, C_{3}\right)$ is important due to the calculation time of the simulation. Too dense a mesh will cause an unacceptably long computation time. When the grid is too sparse the results may not be sufficient. The problem of selecting mesh density has been solved by carrying out the calculation in two steps. After carrying out preliminary tests of numerical simulation, the mesh density in the first step is defined arbitrarily as: $C_{1}$ varies in the range of $0-100$ with step 2, $C_{2}$ and $C_{3}$ varies in the range of $0-8000$ with step 200. The second step is to increase the mesh density but only in the places where the matching function $\varphi$ has reached the highest value in the calculations carried out in the first step. After completing the calculation, $\varphi$ as the function of parameters $C_{1}$ and $C_{2}$ can be drawn. To better visualize the relationship, function $\varphi$ in Fig. 5 is presented in the eighth power.

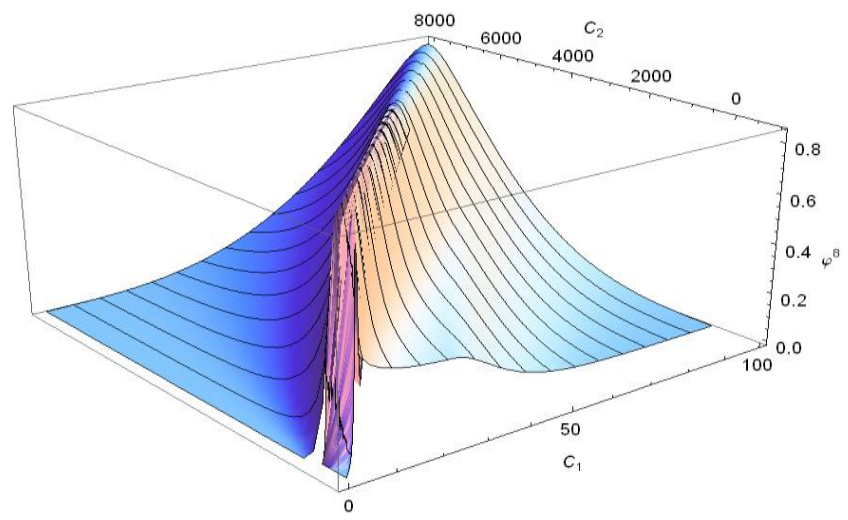

Fig. 5 Matching function $\varphi^{8}\left(C_{1}, C_{2}\right)$

The maximum values of the fit (forming the surface ridge) are arranged in a straight line. One can speak of a linear relationship between $C_{1}$ and $C_{2}$ when you take into account the best matching points. Taking into account 200 points of the best fit (ridge of the surface) this relationship can be drawn as shown in Fig. 6. $\bar{\varphi}$ in Fig. 6 is the average value of the matching function for 200 points of the best fit.

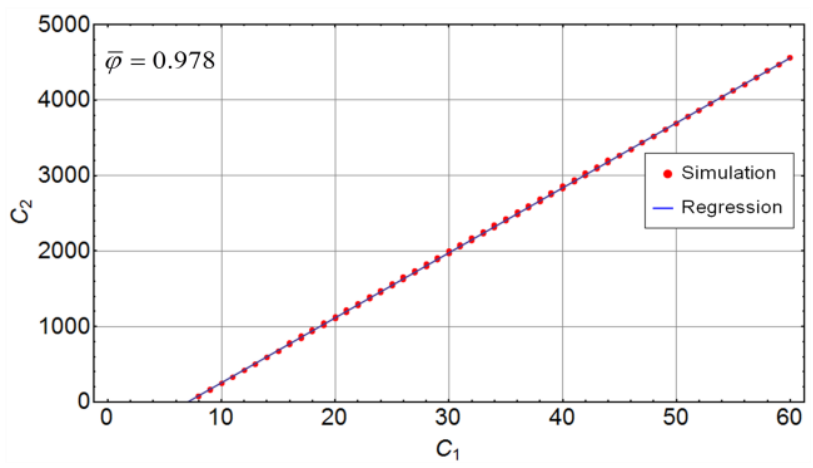

Fig. 6 Linear regression $C_{2}=f\left(C_{1}\right)$

The linear regression function between $C_{1}$ and $C_{2}$ takes form:

$$
C_{2}=(86.07 \pm 0.06) \frac{1}{\mathrm{~s}} C_{1}+(-606.22 \pm 2.06) \frac{\mathrm{Nm}}{\mathrm{rad}},
$$

with the coefficient of determination: $r^{2}=0.999916$. The linear regression result (16) shows a strong linear relation- 
ship between $C_{1}$ and $C_{2}$. In the same way relationship $\varphi\left(C_{2}, C_{3}\right)$ can be determined. To better visualize the relationship, function $\varphi$ in Fig. 7 is presented in the thirtieth power.

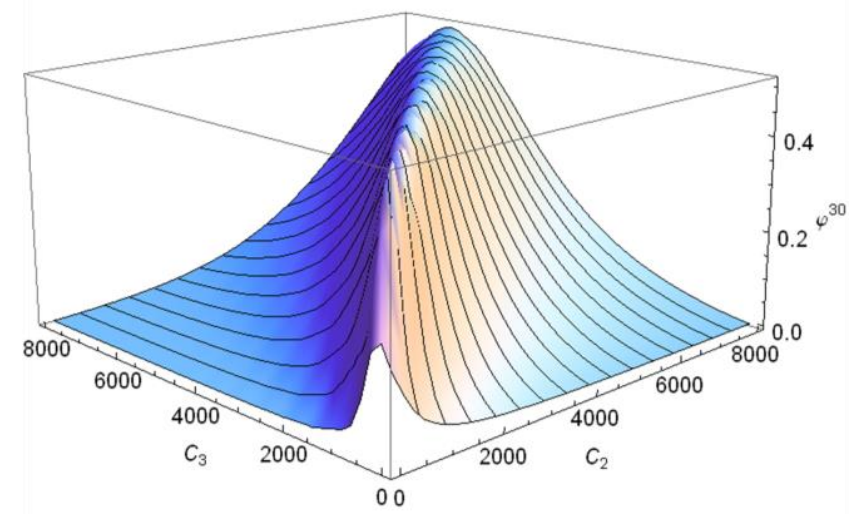

Fig. 7 Matching function $\varphi^{30}\left(C_{2}, C_{3}\right)$

Taking into account 200 points of the best fit, the relationship can be drawn as shown in Fig. 8.

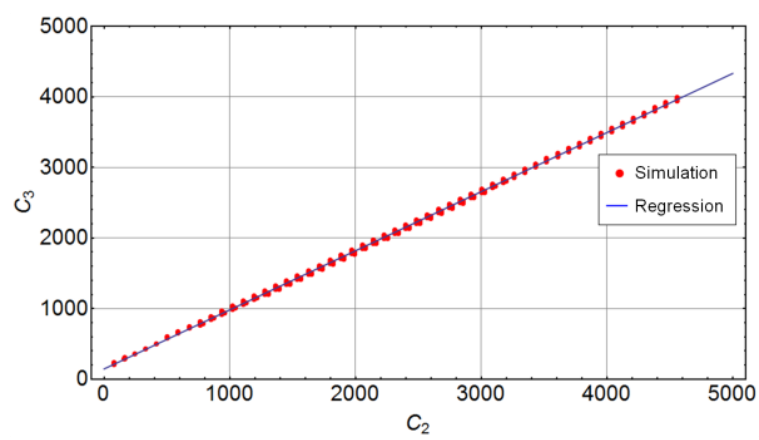

Fig. 8 Linear regression $C_{3}=f\left(C_{2}\right)$

The linear regression function between $C_{2}$ and $C_{3}$ takes the form:

$C_{3}=(0.837 \pm 0.001) \mathrm{s} C_{2}+(145.04 \pm 3.83) \mathrm{Nms} / \mathrm{rad}$,

with the coefficient of determination: $r^{2}=0.99939$. From the above regression results it can be concluded that three unknown parameters $C_{1}, C_{2}, C_{3}$ in function (13) can be expressed by one unknown parameter $C_{1}$.

To determine the unknown parameter $C_{1}$, the relationship between the maximum value of $\varphi$ for each $C_{1}$ should be calculated. This relationship, i.e. $\varphi_{\max }=\operatorname{Max}\left[\varphi\left(C_{1}\right)\right]$, is presented in Fig. 9. The resulting data can be approximated using nonlinear regression. As can be seen in Fig. 9, the approximating function should quickly reach the saturation point. The family of functions having such properties are logistics functions. In this case, the logistic function reaches a saturation level too slowly. The better solution is to use a modified logistic function which takes the form:

$$
\varphi_{\max }=\frac{a}{1+e^{-b C_{1}^{2}-c}} .
$$

Nonlinear regression results for function (18) take the following values: $a=0.9783 \pm 0.0005, c=-0.127 \pm 0.012$,

$b=(0.098 \pm 0.001)(\mathrm{rad} / \mathrm{Nms})^{2}$.

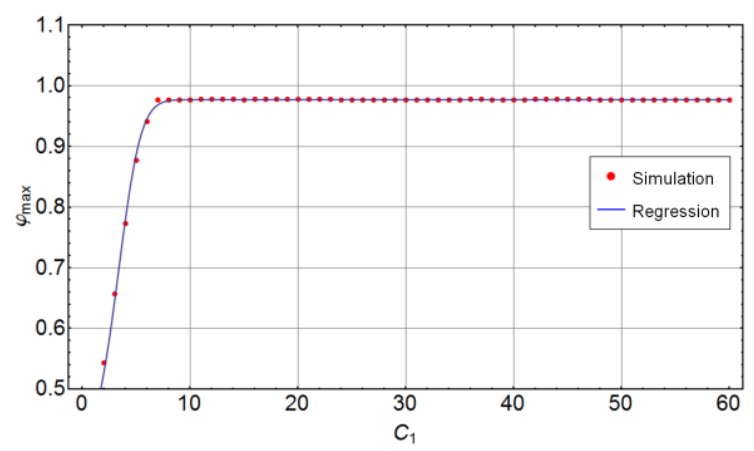

Fig. 9 Nonlinear regression $\varphi_{\max }\left(C_{1}\right)$

To specify the unknown parameter $C_{1}$ from function (18), such $C_{1}$ should be taken for which function $\varphi_{\max }$ has reached a saturation point (parameter $a$ in Eq. (18)). In theory, of course, a saturation point is reached at infinity. In practice, it can be assumed this will happen in a situation where $\varphi_{\max } \approx a$. In these calculations it was assumed that $\varphi_{\max }$ has reached a saturation point when $\varphi_{\max }=0.999 a$. When $\varphi_{\max }=0.999 a$, the matching function $\varphi$ has a sufficiently high value, while the rider's energy consumption is low. Thus, $C_{1}$ value is a value for which $\varphi_{\max }=0.999 a$. According to (18):

$$
C_{1}=\sqrt{-\frac{\operatorname{Ln}\left(0.001001 e^{c}\right)}{b}}=8.45 \frac{\mathrm{Nms}}{\mathrm{rad}} .
$$

Using parameter $C_{1}$ described by (19) and formulas (16), (17), the rider - vehicle interaction $T_{u}$ given by (13) can be determined. It takes the form:

$$
\begin{aligned}
T_{u} & =8.45 \frac{\mathrm{mNs}}{\mathrm{rad}}\left(\dot{\theta}_{S E T}-\dot{\theta}\right)+121.09 \frac{\mathrm{mN}}{\mathrm{rad}}(\alpha-\beta)+ \\
& +246.72 \frac{\mathrm{mNs}}{\mathrm{rad}}(\dot{\alpha}-\dot{\beta}) .
\end{aligned}
$$

The comparison of the experimental results (see Fig. 2) with the numerical simulation results, when interaction torque $T_{u}$ is described by (20), is shown in Fig. 10.

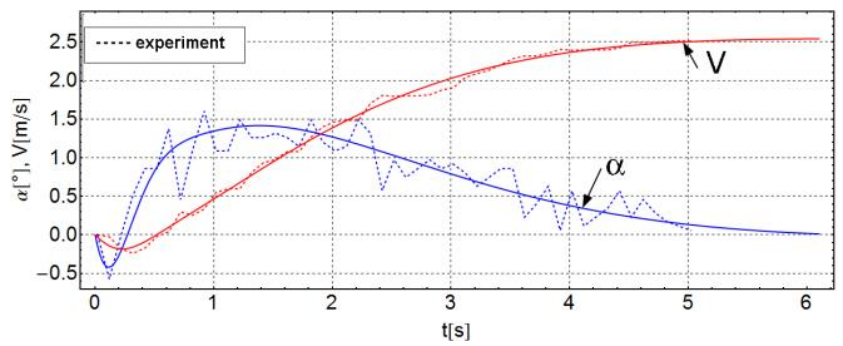

Fig. 10 Comparison of the results for experiment no. 1

The solid lines describes the numerical data ( $\alpha$-platform inclination, $V$ - vehicle velocity). The dashed lines describe the experimental data. The matching functions for the velocity of vehicle is equal to: $\varphi_{V}=0.98$, and for the platform inclination it is: $\varphi_{a}=0.91$. The matching function results confirm the correctness of the hypothesis about the interaction form described by Eq. (13). It is worth emphasizing, that the matching function $\varphi$ was defined based on the vehicle velocity only. Even so, the matching function results for the platform inclination has a high value, and it can be seen in Fig. 10. Angle $\beta$, which describes the rider inclination (Fig. 4), was not measured in 
the experiment. However, it was determined in the computer simulation. To illustrate the rider's behaviour, it is better to show the plot $\beta$ - $\alpha$, that means the rider's inclination with respect to the platform:

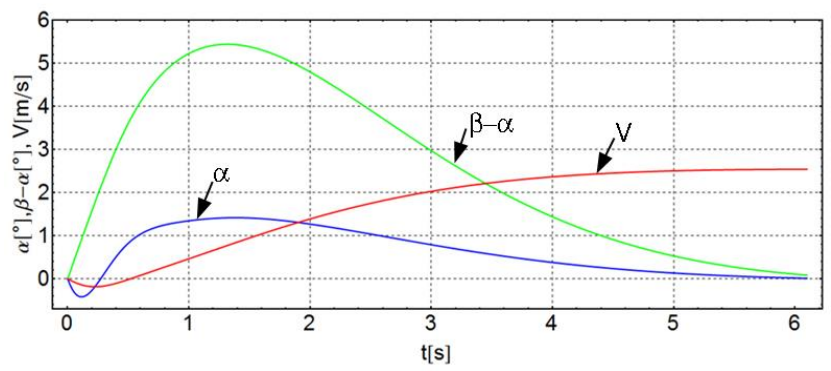

Fig. 11 Simulation results for experiment no. 1
The matching functions (15) were calculated for all thirty experiments (see Section 2). For each of them an analysis of the determination of parameters $C_{1}, C_{2}, C_{3}$ was calculated. Detailed results of the analysis are presented in Table 2. Table 2 presents the results obtained for 30 experiments. Column "No." specifies the number of the experiment, $r^{2}$ is a coefficient of determination for linear regression, $T$ - the vehicle acceleration time measured in the experiment and $V_{T}$ - the desired final velocity measured in the experiment. The average matching functions for thirty experiments take the following values: $\bar{\varphi}_{V}=0.97$, $\bar{\varphi}_{\alpha}=0.91$.

Linear regression and experimental results

Table 2

\begin{tabular}{|c|c|c|c|c|c|c|c|c|c|c|c|}
\hline \multirow[b]{2}{*}{ No. } & \multirow[b]{2}{*}{$\begin{array}{c}C_{1}, \\
\mathrm{Nms} / \mathrm{rad} \\
\end{array}$} & \multicolumn{4}{|c|}{$C_{2}=f_{1}\left(C_{1}\right)=a_{1} C_{1}+b_{1}$} & \multicolumn{4}{|c|}{$C_{3}=f_{2}\left(C_{2}\right)=a_{2} C_{2}+b_{2}$} & \multirow[b]{2}{*}{$T, \mathrm{~s}$} & \multirow[b]{2}{*}{$V_{T}, \mathrm{~m} / \mathrm{s}$} \\
\hline & & $\begin{array}{l}a_{1}, \\
1 / \mathrm{s}\end{array}$ & $\begin{array}{c}b_{1}, \\
\mathrm{Nm} / \mathrm{rad}\end{array}$ & $r^{2}$ & $\begin{array}{c}C_{2}, \\
\mathrm{Nm} / \mathrm{rad}\end{array}$ & $\begin{array}{c}a_{2} \\
\mathrm{~s}\end{array}$ & $\begin{array}{c}b_{2} \\
\mathrm{Nms} / \mathrm{rad}\end{array}$ & $r^{2}$ & $\begin{array}{c}C_{3}, \\
\mathrm{Nms} / \mathrm{rad}\end{array}$ & & \\
\hline 1 & 8.45 & 86.07 & -606.20 & 0.999 & 121.09 & 0.84 & 145.00 & 0.999 & 246.72 & 5.10 & 2.51 \\
\hline 2 & 9.21 & 75.28 & -603.50 & 0.999 & 89.83 & 0.66 & 32.44 & 0.999 & 91.73 & 5.08 & 2.27 \\
\hline 3 & 9.48 & 74.81 & -601.70 & 0.999 & 107.50 & 0.92 & 186.40 & 0.996 & 285.30 & 4.30 & 2.49 \\
\hline 4 & 8.46 & 82.55 & -600.70 & 0.999 & 97.67 & 0.86 & 148.20 & 0.998 & 232.20 & 5.60 & 2.39 \\
\hline 5 & 10.00 & 68.97 & -599.90 & 0.998 & 89.80 & 0.61 & 3.78 & 0.987 & 58.56 & 5.08 & 2.11 \\
\hline 6 & 9.62 & 72.39 & -601.90 & 0.998 & 94.49 & 0.68 & 42.66 & 0.985 & 106.91 & 4.56 & 2.48 \\
\hline 7 & 9.44 & 74.05 & -595.20 & 0.997 & 103.83 & 0.72 & 54.53 & 0.980 & 129.29 & 4.27 & 2.47 \\
\hline 8 & 9.98 & 70.76 & -599.40 & 0.999 & 106.78 & 0.70 & 55.38 & 0.993 & 130.13 & 4.34 & 2.22 \\
\hline 9 & 9.04 & 78.55 & -599.00 & 0.999 & 111.09 & 0.75 & 83.31 & 0.991 & 166.63 & 4.42 & 2.50 \\
\hline 10 & 10.26 & 68.80 & -593.40 & 0.994 & 112.49 & 0.70 & 45.00 & 0.960 & 123.74 & 4.00 & 2.00 \\
\hline 11 & 12.45 & 57.86 & -592.30 & 0.995 & 128.06 & 0.67 & 29.13 & 0.971 & 114.93 & 3.32 & 1.80 \\
\hline 12 & 8.84 & 80.64 & -607.60 & 0.999 & 105.26 & 0.75 & 91.60 & 0.996 & 170.54 & 4.76 & 2.58 \\
\hline 13 & 11.06 & 62.31 & -593.60 & 0.998 & 95.55 & 0.86 & 145.10 & 0.992 & 227.27 & 3.90 & 2.61 \\
\hline 14 & 8.52 & 81.44 & -602.80 & 0.999 & 91.07 & 0.69 & 46.01 & 0.990 & 108.85 & 5.11 & 2.35 \\
\hline 15 & 9.43 & 74.77 & -602.90 & 0.999 & 102.18 & 0.89 & 168.20 & 0.999 & 259.14 & 5.00 & 2.71 \\
\hline 16 & 9.86 & 72.28 & -597.60 & 0.998 & 115.08 & 0.67 & 31.66 & 0.984 & 108.76 & 4.42 & 2.25 \\
\hline 17 & 10.19 & 66.94 & -598.70 & 0.994 & 83.42 & 0.66 & 28.00 & 0.967 & 83.06 & 4.65 & 2.83 \\
\hline 18 & 9.55 & 71.57 & -594.90 & 0.994 & 88.59 & 0.64 & 8.08 & 0.962 & 64.78 & 4.72 & 2.56 \\
\hline 19 & 9.95 & 71.98 & -601.00 & 0.997 & 115.20 & 0.65 & 26.00 & 0.986 & 100.88 & 4.32 & 2.68 \\
\hline 20 & 9.44 & 72.12 & -604.30 & 0.996 & 76.51 & 0.71 & 62.71 & 0.975 & 117.03 & 4.70 & 2.30 \\
\hline 21 & 10.02 & 72.75 & -608.80 & 0.999 & 120.16 & 0.66 & 38.74 & 0.995 & 118.04 & 4.50 & 2.91 \\
\hline 22 & 10.08 & 70.55 & -604.00 & 0.999 & 107.14 & 0.86 & 159.70 & 0.996 & 251.84 & 4.30 & 3.18 \\
\hline 23 & 11.00 & 64.34 & -602.30 & 0.998 & 105.44 & 0.83 & 130.00 & 0.990 & 217.52 & 4.01 & 2.45 \\
\hline 24 & 10.66 & 62.28 & -595.30 & 0.998 & 68.60 & 1.08 & 271.20 & 0.991 & 345.29 & 3.80 & 2.75 \\
\hline 25 & 9.41 & 71.78 & -595.60 & 0.997 & 79.85 & 0.89 & 155.00 & 0.981 & 226.07 & 4.30 & 2.48 \\
\hline 26 & 9.84 & 72.35 & -606.30 & 0.999 & 105.62 & 0.73 & 76.13 & 0.995 & 153.24 & 4.52 & 2.63 \\
\hline 27 & 8.89 & 78.08 & -604.20 & 0.999 & 89.93 & 0.83 & 132.30 & 0.995 & 206.94 & 4.80 & 2.68 \\
\hline 28 & 10.10 & 68.51 & -598.70 & 0.997 & 93.25 & 0.68 & 37.68 & 0.982 & 101.09 & 4.40 & 2.62 \\
\hline 29 & 10.75 & 65.05 & -593.70 & 0.998 & 105.59 & 0.70 & 41.51 & 0.987 & 115.42 & 3.93 & 2.53 \\
\hline 30 & 10.95 & 62.70 & -597.80 & 0.997 & 88.77 & 0.74 & 77.55 & 0.982 & 143.24 & 4.20 & 2.47 \\
\hline
\end{tabular}


The comparison of the experimental results with the numerical simulation results for a few selected experiments is shown in Fig. 12 and Fig. 13.

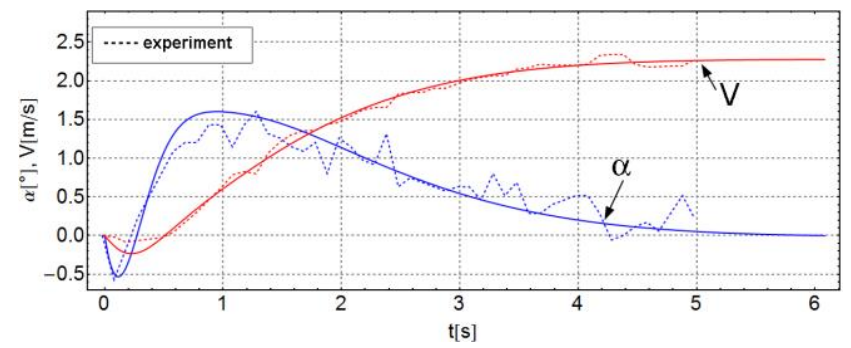

Fig. 12 Comparison of the results for experiment no. 2

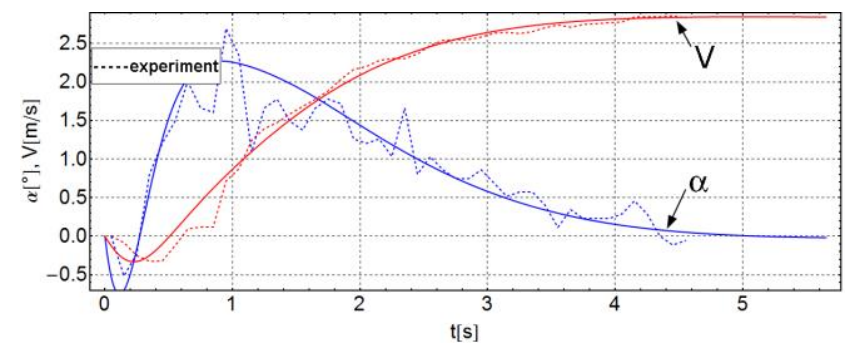

Fig. 13 Comparison of the results for experiment no. 17

\section{Generalized form of interaction}

The designated model, however, has some disadvantages - it is not a general model. To describe each of the thirty experiments, parameter $C_{1}$ and function $C_{2}=f\left(C_{1}\right)$ and $C_{3}=f\left(C_{2}\right)$ are required. The interaction described by equation (13) explicitly depends on the desired final wheel speed, and thus the desired final linear velocity of the vehicle. It is a natural assumption because the user's goal is acceleration to the desired velocity. It is obvious that the user can reach the desired final velocity at different times, so torque $T_{u}$ should explicitly depend on the time needed to obtain this speed. To check if torque $T_{u}$ depends on the acceleration time $T$, the relationship between the designated parameter $C_{1}$ and vehicle acceleration time $T$ should be examined. This relationship is shown in Fig. 14.

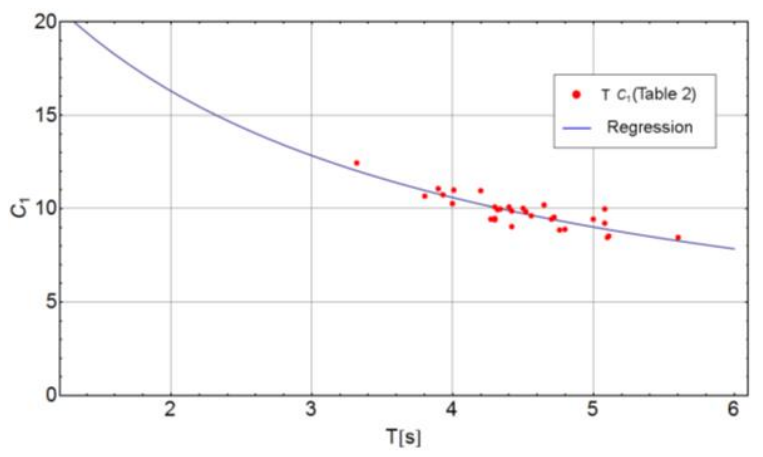

Fig. 14 The relationship $C_{1}=f(T)$

The results presented in Fig. 14 show, that parameter $C_{1}$ can be expressed as a function of acceleration time $T$. If the rider intends to accelerate to the desired final velocity in a long time, torque $T_{u}$, and hence parameters $C_{1}$, should be small. In another words, parameter $C_{1}$ should be inversely proportional to time $T$. Therefore, the function approximating the relationship between $C_{1}$ and $T$ takes the form:

$$
C_{1}(T)=\frac{a_{C T}}{b_{C T}+T}
$$

where $a_{C T}=(60.61 \pm 6.58) \frac{\mathrm{Nms}^{2}}{\mathrm{rad}}, \quad b_{C T}=(1.72 \pm 0.66) \mathrm{s}$.

Looking at the results in Table 2, it can be concluded that coefficients $b_{1}$ for the thirty experiments differ very little from each other and can be regarded (with a good approximation) as equal. It turns out that there is a fairly strong relationship between parameters $C_{1}$ and $a_{1}$ contained in Table 2. This relationship is shown in Fig. 15.

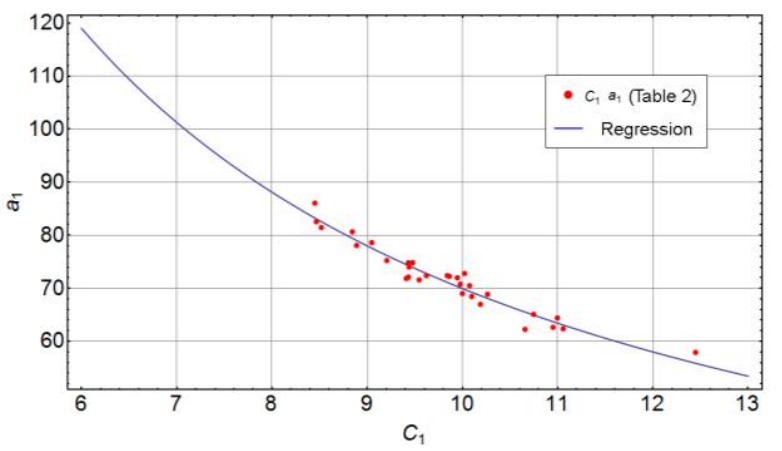

Fig. 15 The relationship $a_{1}=f\left(C_{1}\right)$

Based on the graphical presentation of the data shown in Fig. 15, it can be assumed that the function approximating the relationship between $C_{1}$ and $a_{1}$ takes the form:

$$
a_{1}\left(C_{1}\right)=\frac{a_{a C}}{b_{a C}+C_{1}}
$$

where

$a_{a C}=(678.71 \pm 31.95) \frac{\mathrm{Nm}}{\mathrm{rad}}, \quad b_{a C}=(-0.30 \pm 0.44) \frac{\mathrm{Nms}}{\mathrm{rad}}$

As a result, parameter $C_{2}$ in Eq. (13) can be written as:

$$
C_{2}=f_{1}\left(C_{1}\right)=a_{1} C_{1}+\bar{b}_{1}=\frac{a_{a C}}{b_{a C}+C_{1}} C_{1}+\bar{b}_{1},
$$

where $\bar{b}_{1}$ is mean value of parameter $b_{1}$. Substituting $C_{1}$ from Eq. (21) into Eq. (23) is obtained as follows:

$$
C_{2}=\frac{a_{a C} a_{C T}}{a_{C T}+b_{a C}\left(T+b_{C T}\right)}+\bar{b}_{1}
$$

According to Eqs. (21) and (24), parameters $C_{1}$ and $C_{2}$ can be represented as a function of acceleration time $T$. Unfortunately, parameters of function $C_{3}=f_{2}\left(C_{2}\right)$ listed in Table 2, did not show any dependence on time $T$ or parameters $C_{1}$ or $C_{2}$. In this case, generalization consists in taking the average values of coefficients $a_{2}$ and $b_{2}$ obtained for the thirty experiments. Parameter $C_{3}$ in Eq. (13) can be written as:

$$
C_{3}=f_{2}\left(C_{2}\right)=\bar{a}_{2} C_{2}+\bar{b}_{2} \text {. }
$$

Substituting $C_{2}$ from Eq. (24) into Eq. (25) is obtained as follows: 


$$
C_{3}=\bar{a}_{2}\left(\frac{a_{a C} a_{C T}}{a_{C T}+b_{a C}\left(T+b_{C T}\right)}+\bar{b}_{1}\right)+\bar{b}_{2} .
$$

According to Eqs. (21), (24), (26) and (13) the generalized form of interaction between the vehicle and its rider is expressed as follows:

$$
\begin{aligned}
& T_{u}=\frac{a_{C T}}{b_{C T}+T}\left(\dot{\theta}_{S E T}-\dot{\theta}\right)+\left(\frac{a_{a C} a_{C T}}{a_{C T}+b_{a C}\left(T+b_{C T}\right)}+\bar{b}_{1}\right) \times \\
& \times(\alpha-\beta)+\left(\bar{a}_{2}\left(\frac{a_{a C} a_{C T}}{a_{C T}+b_{a C}\left(T+b_{C T}\right)}+\bar{b}_{1}\right)+\bar{b}_{2}\right) \times \\
& \times(\dot{\alpha}-\dot{\beta}) .
\end{aligned}
$$

Substituting the numerical values of parameters, Eq. (27) takes the form:

$$
\begin{aligned}
T_{u} & =\frac{60.61}{1.72 \mathrm{~s}+T} \frac{\mathrm{Nms}^{2}}{\mathrm{rad}}\left(\dot{\theta}_{\text {SET }}-\dot{\theta}\right)+ \\
& +\left(\frac{41138.67}{60.10-0.30 \frac{1}{\mathrm{~s}} T}-600.11\right) \frac{\mathrm{Nm}}{\mathrm{rad}}(\alpha-\beta)+ \\
& +\left(\frac{31016.11}{60.10-0.30 \frac{1}{\mathrm{~s}} T}-367.35\right) \frac{\mathrm{Nms}}{\mathrm{rad}}(\dot{\alpha}-\dot{\beta}) .
\end{aligned}
$$

It should be emphasized that the general form of interaction described by Eqs. (27) or (28) has been designated for a particular vehicle with a particular user and for a specific control law of vehicle motors. Changing the physical properties of the vehicle (e.g. mass of the platform or radius of the wheels) or the rider (e.g. their mass) will cause that the designated numerical values in Eq. (28) will not be appropriate for a system with the altered physical properties. Changing the motor control law described by Eq. (12) will have the same effect. In order to express all the numerical parameters of Eq. (28) as the physical properties of the system, a series of experiments for vehicles and users with different values of physical properties occurring in the mathematical model of the vehicle must be performed. Then, you need to carry out an extensive statistical analysis.

The comparison of the experimental results with the numerical simulation results using a general form of interaction for a few selected experiments is shown in the graphs in Fig. 16, Fig. 17 and Fig. 18.

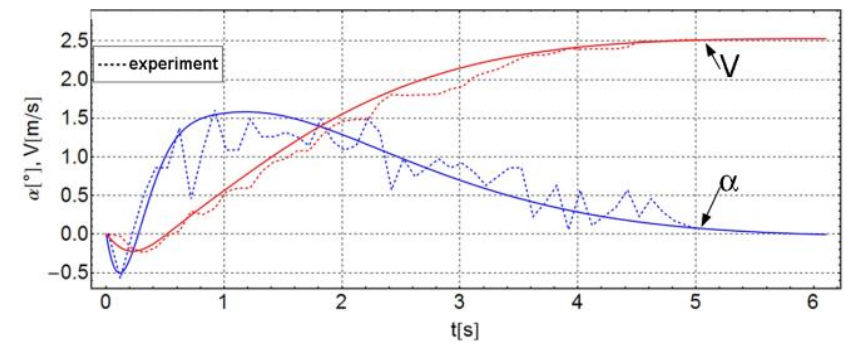

Fig. 16 Comparison of the results for experiment no. 1

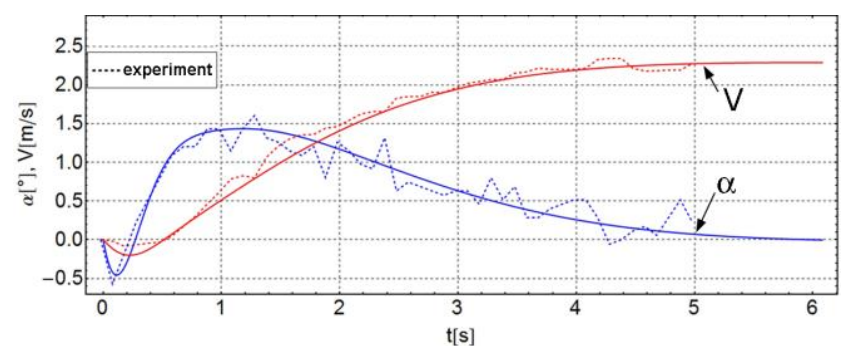

Fig. 17 Comparison of the results for experiment no. 2

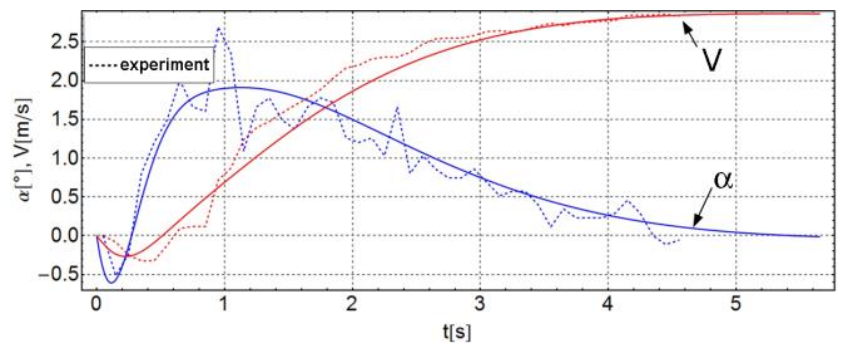

Fig. 18 The comparison of the results for experiment no.

The average matching functions for thirty experiments take the values: $\bar{\varphi}_{V}=0.957, \bar{\varphi}_{\alpha}=0.91$.

\section{Conclusions}

Determination of the mathematical model of interaction between the vehicle and its rider, consisted of several stages. Firstly, an experiment was carried out. Secondly, the mathematical model of the system was created. Thirdly, the hypothesis about the character of interaction was postulated. Next, the comparison method between the simulation and experimental data was developed. Finally, the quantitative and qualitative description of interaction and its generalized form was designated. The results presented on the graphs (Figs. 16, 17, 18) show, that the generalized form of interaction between the TWSBV and its rider can be recognized, with high probability, as correct. This is also confirmed by the results of the matching functions. Of course, if parameters $C_{1}, C_{2}, C_{3}$ in Eq. (13) are unique for each experiment, the results of the matching functions are better than in the general case. However, the difference is not big, and the benefit of generalization is enormous. The generalized description of the interaction depends explicitly only on two variables (excluding, of course, the state vector). These variables are the desired final velocity and the time after which this velocity will be achieved. As a result of the analysis, the previously unknown torque $T_{u}$ applied by the user in the ankle joint was described in this way. Therefore, the mathematical model of the vehicle-rider system became more realistic than the commonly used two-wheeled inverted pendulum model.

\section{Acknowledgements}

I would like to thank my supervisor professor Ewa Pawluszewicz for discussion and constructive comments.

The work was supported by the Bialystok University of Technology grant No. S/WM/1/2012. 


\section{References}

1. Grasser, F.; D'Arrigo, A.; Comlombi, S.; et al. 2002. Joe: A mobile inverted pendulum, IEEE Trans., Electronics 49-1: 107-114. http://dx.doi.org/10.1109/41.982254.

2. Pathak, K.; Franch, J.; Agrawal, S.K. 2005. Velocity and position control of a wheeled inverted pendulum by partial feedback linearization, IEEE Transactions on Robotics 21-3: 505-513.

http://dx.doi.org/10.1109/TRO.2004.840905.

3. Baloh, M.; Parent, M. 2003. Modeling and Model Verification of an intelligent self balancing twowheeled vehicle for an autonomous urban transportation system, The Conference on Computational Intelligence, Robotics, and Autonomous Systems, Singapore.

4. Lin, S.C.; Tsai, C.C.; Luo, W.L. 2007. Adaptive neural network control of a self-balancing two-wheeled scooter, Industrial Electronics Society, 2007. IECON 2007. 33rd Annual Conference of the IEEE: 868-873. http://dx.doi.org/10.1109/IECON.2007.4460153.

5. Azizan, H.; Jafarinasab, M.; Behbahani, S.; et al. 2010. Fuzzy control based on LMI approach and fuzzy interpretation of the rider input for two wheeled balancing human transporter, 8th IEEE International Conference on Control and Automation (ICCA): 192-197. http://dx.doi.org/10.1109/ICCA.2010.5524327.

6. Pinto, L.J.; Dong-Hyung, K.; Ji Yeong L.; et al. 2012. Development of a Segway robot for an intelligent transport system, International Symposium on System Integration: 710-715. http://dx.doi.org/10.1109/SII.2012.6427308.

7. Castro, A.; Adams, C.; Singhose, W.E. 2013. Dynamic response characteristics of a two-wheeled inverted-pendulum transporter, in Proc. CDC: 1532-1537. http://dx.doi.org/10.1109/CDC.2013.6760100.

8. Huang, C.-H.; Wang, W.-J.; Chiu, C.-H. 2011. Velocity control realisation for a self-balancing transporter, IET Control Theory \& Applications 5-13: 15511560 . http://dx.doi.org/10.1049/iet-cta.2010.0530.

9. Mohtasib, A.M.; Shawar, M.H. 2013. Self-balancing two-wheel electric vehicle (STEVE), 9th International Symposium on Mechatronics and its Applications: 1-8. http://dx.doi.org/10.1109/ISMA.2013.6547384.

Ciężkowski, M.; Pawluszewicz, E. 2015. Determination of interactions between two-wheeled selfbalancing vehicle and its rider, 20th International Conference on Methods and Models in Automation and Robotics MMAR'2015: 851-855.

10. Bjorck, A.; Dahlquist, G. 1974. Numerical Methods, Prentice-Hall.

11. Winter, D.A.; Patla, A.E.; Prince, F.; et al. 1998. stiffness control of balance in quiet standing, Journal of
Neurophysiology 80-3: 1211-1221.

http://jn.physiology.org/content/80/3/1211.

12. Loram, I.D.; Kelly, S.M.; Lakie, M. 2001. Human balancing of an inverted pendulum is sway size controlled by ankle impedance?, Journal of Physiology 532-3: 879-891.

http://www.ncbi.nlm.nih.gov/pubmed/11313453. http://dx.doi.org/10.1111/j.1469-7793.2001.0879e.x.

13. Landau, L.D.; Lifszyc, J.M. 1976. Mechanics 3rd ed., Oxford, England Pergamon Press.

14. Bryson, A.E.; Ho, Y.C. 1969. Applied Optimal Control, Blaisdell, Waltham, MA.

15. Boyd, S.; Barratt, C. 1991. Linear Controller Design Limits of Performance,Prentice-Hall.

16. Ciężkowski, M.; Mystkowski, A. 2015. Projekt dwukołowego samobalansującego pojazdu, Przegląd Elektrotechniczny 2: 147-152, (in polish). http://pe.org.pl/articles/2015/2/34.pdf

17. Papoulis, A.; Pillai, S. 2002. Probability Random Variables and stochastic Processes, 4th edition, New York McGraw-Hill, 852 p.

\section{Ciężkowski}

\section{METHOD FOR DETERMINATION OF INTERACTION BETWEEN A TWO-WHEELED SELF-BALANCING VEHICLE AND ITS RIDER}

S u m m a r y

The paper presents a method for determination of interaction between a two-wheeled self-balancing vehicle and its rider while driving. The presented approach is a new direction of research, because this kind of interaction is usually overlooked in simulation and analytical studies whose aim is to determine and verify the vehicle control method. The proposed method is based on the comparison of experimental results with the computer simulation results. The mathematical model of the vehicle-rider system used in the simulation includes a postulated qualitative mathematical form of interaction with unknown coefficients (in practice, they estimate parameters of the rider). These unknown coefficients have been obtained by designation of the matching function between the experimental data and simulation data. Based on these coefficients, the generalized form of interaction between a two-wheeled self-balancing vehicle and its rider has been found.

Keywords: interaction, two-wheeled self-balancing vehicle, modelling, identification.

Received October 05, 2015 Accepted September 28, 2016 